\title{
Prevalence and Associated Symptoms of Helicobacter pylori Infection among Schoolchildren in Kassala State, East of Sudan
}

\author{
Mohammed Abbas, ${ }^{1,2}$ Faiza A. Sharif, ${ }^{3}$ Shamselfalah M. Osman, ${ }^{2,4}$ Abdallah M. Osman, ${ }^{2}$ \\ Sulieman M. El Sanousi, ${ }^{5}$ Mamoun Magzoub, ${ }^{6}$ and Mutasim E. Ibrahim $\oplus^{7}$ \\ ${ }^{1}$ Department of Pediatrics, College of Medicine, University of Bisha, Bisha, Saudi Arabia \\ ${ }^{2}$ Department of Pediatrics, Faculty of Medicine \& Health Sciences, University of Kassala, Kassala, Sudan \\ ${ }^{3}$ Department of Microbiology, Faculty of Medicine \& Health Sciences, University of Kassala, Kassala, Sudan \\ ${ }^{4}$ Department of Pediatrics, King Saud Medical City, Riyadh, Saudi Arabia \\ ${ }^{5}$ Department of Microbiology, Faculty of Veterinary Medicine, University of Khartoum, Khartoum, Sudan \\ ${ }^{6}$ Department of Parasitology, Faculty of Medical Laboratory Science, International University of Africa, Khartoum, Sudan \\ ${ }^{7}$ Department of Clinical Microbiology and Parasitology, College of Medicine, University of Bisha, Bisha, Saudi Arabia
}

Correspondence should be addressed to Mutasim E. Ibrahim; mutasimhadi87@hotmail.com

Received 30 August 2017; Revised 10 November 2017; Accepted 17 December 2017; Published 15 January 2018

Academic Editor: Subhada Prasad Pani

Copyright (C) 2018 Mohammed Abbas et al. This is an open access article distributed under the Creative Commons Attribution License, which permits unrestricted use, distribution, and reproduction in any medium, provided the original work is properly cited.

\begin{abstract}
This study aimed to determine the prevalence of $H$. pylori infections among schoolchildren and investigate the associations between H. pylori seropositivity and existence of gastrointestinal symptoms. Methods. A prospective cross-sectional study was conducted during a period from January to December 2012 at Kassala state, east of Sudan. Schoolchildren from different primary schools were enrolled in the study. Sociodemographic characteristics and gastrointestinal symptoms were recorded from each child. A rapid immunochromatographic test was performed for the detection of $H$. pylori IgG antibodies. Data on patient demographic characteristics, clinical diagnosis, and findings of $H$. pylori infection were analyzed by simple descriptive statistics. Results. Among 431 schoolchildren, $H$. pylori seropositivity was found to be $21.8 \%$. The majority of children $(79 ; 84 \%)$ had BMI below the normal range. The most frequent symptoms associated with $H$. pylori infections were nausea $(25.5 \%)$, followed by gastric pain $(24.5 \%)$ and heart pain (20.2\%). There were statistically significant differences in $H$. pylori seropositivity between boys and girls $(p=0.003)$. Conclusions. The prevalence of $H$. pylori infection among schoolchildren in Kassala city has been documented. Although the majority of the disease was associated with several gastrointestinal symptoms, the role of infection in the etiology of abdominal symptoms needs further investigations.
\end{abstract}

\section{Introduction}

Helicobacter pylori (H. pylori) is a gram-negative bacterium colonizing human stomach and associated with numerous gastrointestinal diseases [1]. It is quite a frequent infection all over the world; more than half of the population in both developed and developing countries are infected with this microorganism $[2,3]$. Most of the people acquire $H$. pylori infection during their early childhood [4]. H. pylori has been reported as a common cause of chronic gastritis, peptic ulcer diseases, and gastric cancer in adults [5].
Most of $H$. pylori infections usually are symptomless and without clinical manifestation, particularly in poor communities [6]. However, signs and symptoms associated with the disease are primarily due to gastric or peptic ulcer illness or duodenal inflammation. Furthermore, other symptoms such as nausea, vomiting, and abdominal pain may be attributed to other gastrointestinal diseases [7].

H. pylori infection developed during early childhood is considered as a significant risk factor for gastric carcinoma in the adult individual [8]. It is well known that $H$. pylori 
infection among children is associated with several extragastric diseases, including growth reduction, iron-deficiency anemia, and idiopathic thrombocytopenic purpura $[1,7]$. Schoolchildren in developing countries are at-risk group due to several factors including low socioeconomic status, poor quality of drinking water, overcrowding, poor personal and environmental hygiene, and food contamination $[2,9]$.

Screening for the serum IgG antibody to $H$. pylori is a practical method for diagnosing $H$. pylori infection in children. However, measurements of the H. pylori IgG antibody are useful for monitoring treatment of $H$. pylori infection because of its high sensitivity and ease of performance $[10,11]$. Therefore, this study aimed to determine the prevalence of $H$. pylori infections among schoolchildren in Kassala and to investigate the associations between $H$. pylori seropositivity and presence of gastrointestinal symptoms.

\section{Materials and Methods}

A prospective randomized cross-sectional study was conducted during a period from January to December 2012 at Kassala state, east of Sudan (about 500 kilometers from Khartoum capital). Kassala has a population of 180.000 of different ethnicity and Sudanese tribes [12]. The study population was comprised of schoolchildren, those who were selected from various primary schools in Kassala city.

2.1. Samples and Data Collection. A structured questionnaire has been developed to obtain sociodemographic characteristics and gastrointestinal symptoms. Filling of each questionnaire form was achieved by the authors (pediatricians) during their conduction of clinical examinations. Moreover, the body mass index (BMI) was obtained (weight in $\mathrm{kg} / \mathrm{height}$ in $\mathrm{m} 2$ ) and the schoolchildren were classified as being underweight, healthy, and overweight. Then about $5 \mathrm{ml}$ of blood sample was collected from each child in a plain container for detection of $H$. pylori antibodies. The collected samples were transported to the laboratory, and then sera were separated by centrifugation at $15.000 \mathrm{rpm}$ for 10 minutes and stored at $-20^{\circ} \mathrm{C}$ until being used for serological tests. Children under antibiotics treatment for the last four weeks, with the illness of the liver, renal, pancreatic, or parasitic infections, or evidence of any other viral or bacterial infections were excluded from the study. The study was approved by the ethical clearance committee, Ministry of Health, Kassala state, Sudan. Written consent was obtained from each participant or their parents or guardians after informing them about the importance of the study.

2.2. Screening of $H$. pylori IgG Antibodies. The IgG anti$H$. pylori antibody in serum was detected using a Hexagon H. pylori commercial immunochromatographic test kit (HUMAN Gesellschaft für Biochemica und Diagnostica $\mathrm{mbH}$, Germany) with a high sensitivity (96\%) and specify $(99 \%)$ as per manufacturer's specification. The procedure followed the manufacturer's instructions. In brief, $25 \mu \mathrm{L}$ of serum sample was added to the sample well of the strep using automatic pipette followed by adding 3 drops of buffer.

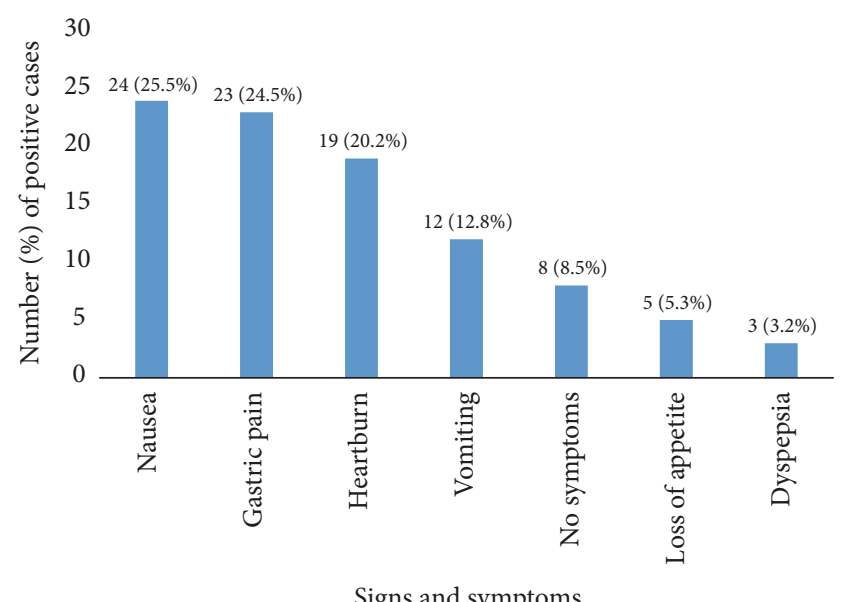

FIGURE 1: Frequency of symptoms associated with $H$. pylori infection among schoolchildren.

The result was read and interpreted macroscopically after 15 minutes. Positive and negative controls were run simultaneously. Positive results were specified by two pink/red bands (control line and test line), seen in the result window of the test cassette, while a negative result was interpreted when only one pink/red band was observed in the control window. In case of no pink/red color developed in control and test line, the test cassette was invalid and the sample under analysis was repeated.

2.3. Data Analysis. Statistical Package for Social Sciences program (SPSS Inc., Chicago, IL, USA) version 16 was used for data entry and for analysis of the patients demographic characteristics; clinical diagnosis and findings of $H$. pylori infection were analyzed by simple descriptive statistics. Chi-square test was used to compare every two variables. A $p$ value lower than 0.05 was statistically significant.

\section{Results}

A total of 431 children (217 boys and 214 girls) from different primary schools were enrolled in the study. The ages of the schoolchildren ranged from 6 to 18 years (mean 11.78 years + 2.035 (SD)).

H. pylori seropositivity was found to be $21.8 \%$ (94/431). The majority of the infected children $(84 \%$; $79 / 94)$ had BMI below the normal range. Of the 94 positive cases, most of them were boys 60 (27.7\%), whereas $34(15.9 \%)$ were girls. There were statistically significant differences in $H$. pylori seropositivity between boys and girls $(p=0.003)$ (Table 1).

As shown in Figure 1, the most common symptoms associated with $H$. pylori infections were nausea (25.5\%), followed by gastric pain (24.5\%) and heart pain (20.2\%).

There was no significant difference of $H$. pylori seropositivity between the all age groups of children (Table 2). 
TABLE 1: Gender-dependent frequency of $H$. pylori infection among schoolchildren (aged between 6 and 18 years).

\begin{tabular}{lccc}
\hline Gender & Total number & Number (\%) of positive cases & $p$ value \\
\hline Boy & 217 & $60(27.7)$ & 0.003 \\
Girl & 214 & $34(15.9)$ & 0 \\
\hline
\end{tabular}

TABLE 2: Distribution of $H$. pylori infection among school-aged children according to their age groups at Kassala city, Sudan.

\begin{tabular}{lccc}
\hline Age group & $\begin{array}{c}\text { Total number of cases } \\
(n=431)\end{array}$ & $\begin{array}{c}\text { Number of positive cases } \\
(n=94)\end{array}$ & \% of positive cases \\
\hline Less than 10 years & 53 & 12 & 22.6 \\
10 to 12 years & 228 & 48 & 21.1 \\
13 to 15 years & 137 & 31 & 22.6 \\
More the 15 years & 13 & 3 & 23.1 \\
\hline
\end{tabular}

\section{Discussion}

Eradication of $H$. pylori infection has currently become an important concern because it can cause many gastroduodenal disorders [13]. To our best knowledge, this is the first study applying rapid one-step immune-chromatographic assay to screen the presence of $H$. pylori antibody among children in Kassala city, east of Sudan. In the present study, the prevalence of $H$. pylori among schoolchildren was $21.8 \%$. This value is similar to those reported in Iran (19.8\%) [13], but higher than $15.1 \%$ reported in Taiwan [8]. However, our finding was lower than 27.4\% reported in Saudi Arabia [14] and 49\% in Turkey [15]. Reports presented in this study, together with reports of others, indicate that there is quite a variation in prevalence of $H$. pylori infections all around the world. Tsongo et al. mentioned that the difference in results is probably due to the variations in the study population, such as the urban dwellers, and the age and health conditions of the patients [9]. Studies document that low socioeconomic status, sanitary conditions, level of educational background, and rates of immigrant children from the surrounding cities are significant risk factors for $H$. pylori infection among children $[8,16,17]$. Therefore, a proper approach to improve lifestyle, sanitary facilities, and promoting economic and educational status has an impact on the decline in the incidence of $H$ pylori infections $[17,18]$. However, prevention and eradication of $H$. pylori infection by treatment of children have been recommended [19]. The North American and European societies of Pediatric Gastroenterology, Hepatology, and Nutrition guidelines for the management of $H$. pylori infection in children recommend triple therapy as a first-line eradication regimen [20]. This treatment regimen should be with three medications including a proton pump inhibitor combined with amoxicillin and clarithromycin or metronidazole. The guidelines also recommended bismuth salts plus amoxicillin and metronidazole as a second-line therapy. Triple therapy should be administered for 7 to 14 days [19, 20]. However, development of antibiotic resistance strains, patient compliance, reinfection, and environmental factors might lead to treatment failure [18-20]. Therefore, understanding the epidemiologic burden of $H$. pylori infection is essential for implementing eradication strategies [18].
In the present study, the incidence of $H$. pylori infection was common among all age groups of schoolchildren. Worldwide studies determined the prevalence of $H$. pylori among school-aged children. For example, in Yemen, the prevalence of $H$. pylori in the age groups 6-8 and 9-12 years was higher than the other age groups [21]. In Saudi Arabia, the prevalence of infection was higher among students in intermediate school (43.7\%) than those in secondary school (19.4\%) [14]. A previous study on epidemiologic characteristics of $H$. pylori infection among different age groups of schoolchildren reported that the seropositive rates were $11.0 \%$ in 9-12 years age group and $12.3 \%$ in $13-15$ years age group. Additionally, the prevalence of infection was increased by the age [8]. These findings indicated that school-aged children are the most common infected group with $H$. pylori infection with varying frequency. Therefore, establishing of health awareness program at school to enhance children personal hygiene and behavior might decrease the risk of disease.

In the present study, boys were more likely to have $H$. pylori infection in comparison to the girls. This finding in consistent with a survey carried out in Uganda [22]. Likewise, in Brazil, the acquisition of $H$. pylori infection was associated with the male gender [23]. In contrary to these findings, a study carried out in Yemen found that girls were more affected than boys [21]. However, elsewhere studies showed no differences in the prevalence of infection between boys and girls $[15,17]$.

H. pylori infection among children can be either clinically silent or associated with nonspecific sign or symptoms, which are seen in various childhood complaints [24]. In this study, the majority of $H$. pylori infection was associated with nausea $(25.5 \%)$ and gastric pain $(24.5 \%)$. Chong et al. suggested that $H$. pylori infection is more frequently associated with gastritis than with peptic ulcer disease in children and that $H$. pylori gastritis is a cause of recurrent abdominal pain syndrome in children [10]. However, the role of $H$. pylori infection in the etiology of abdominal symptoms remains unclear [25]. Furthermore, a recent study found that $H$. pylori infection is declining in symptomatic children, but it is still a common cause of upper gastrointestinal tract symptoms such as chronic abdominal pain/distress, epigastric pain, nausea, or vomiting [17]. 
H. pylori infection acquired in early childhood might affect growth and appetite, consequently influencing body weight of children $[26,27]$. In the study, most of the positive cases were underweight. A study suggested that $H$. pylori infection hurts the growth of children [5]. Additionally, other authors have noted that a positive association of $H$. pylori infection with BMI was only seen in those aged 15+ years [4]. Recently, Benson et al. determined that the odds of being thin in the 10- to 19-year-old age group were 4.28fold higher (95\% CI 1.48-12.4) if they were H. pylori positive compared with those who were $H$. pylori negative [28]. In contrary, $H$. pylori infection was not associated with overweight/obesity observed from the retrospective study in this Chinese population [29]. Therefore, estimation of factors related to social and family environment may clarify the association between $H$. pylori infections and children's growth [4]. However, several factors can affect children's growth and stature, including nutrition, chronic inflammation, and gastrointestinal diseases [30, 31].

In conclusion, the prevalence of $H$. pylori infection among schoolchildren in this study was $21,8 \%$. We think this was a high infection among these children in the study area. Therefore, prevention and eradication of $H$. pylori infection by treatment of children might be essential. In addition, establishing of health awareness program at school to enhance children personal hygiene and behavior is essential to decrease the risk of infection. Although the majority of $H$. pylori infection is associated with different types of gastrointestinal symptoms, the role of $H$. pylori infection in the etiology of abdominal symptoms remains unclear, thus needing further investigations.

\section{Conflicts of Interest}

The authors declare that there are no conflicts of interest regarding this study.

\section{Acknowledgments}

The authors express their gratitude to Mr. AbdaAzeiz Albadawi, Ministry of Education, at Kassala state for supporting this survey. Their thanks are extended to Drs. Fatima Abass and Safaa Abdallah, for their help and support. Special thanks are due to the schools' administrations and schoolchildren for their participation in this survey.

\section{References}

[1] F.-C. Kuo, C.-Y. Wu, C.-H. Kuo et al., "The utilization of a new immunochromatographic test in detection of Helicobacter pylori antibody from maternal and umbilical cord serum," BioMed Research International, vol. 2014, Article ID 568410, 2014.

[2] R. Darko, A. E. Yawson, V. Osei, J. Owusu-Ansah, and S. Aluze-Ele, "Changing patterns of the prevalence of Helicobacter pylori among patients at a corporate hospital in Ghana," Ghana Medical Journal, vol. 49, no. 3, pp. 147-153, 2015.

[3] A. Dane and T. Gurbuz, "Clinical comparative study of the effects of Helicobacter pylori colonization on oral health in children," Pakistan Journal of Medical Sciences, vol. 32, no. 4, 2016.

[4] M. Kopáčová, J. Bureš, I. Koupil et al., "Body indices and basic vital signs in Helicobacter pylori positive and negative persons," European Journal of Epidemiology, vol. 22, no. 1, pp. 67-75, 2007.

[5] J. Vilchis, X. Duque, R. Mera et al., "Association of Helicobacter pylori infection and height of Mexican children of low socioeconomic level attending boarding schools," The American Journal of Tropical Medicine and Hygiene, vol. 81, no. 6, pp. 1091-1096, 2009.

[6] C. Figueiredo, J. C. Machado, P. Pharoah et al., "Helicobacter pylori and interleukin 1 genotyping: an opportunity to identify high-risk individuals for gastric carcinoma," Journal of the National Cancer Institute, vol. 94, no. 22, pp. 1680-1687, 2002.

[7] B. D. Gold, M. A. Gilger, and S. J. Czinn, "New diagnostic strategies for detection of helicobacter pylori infection in pediatric patients," Journal of Gastroenterology and Hepatology, vol. 10, 7, no. 12, pp. 1-19, 2014.

[8] D.-B. Lin, J.-B. Lin, C.-Y. Chen, S.-C. Chen, and W.-K. Chen, "Seroprevalence of Helicobacter pylori infection among schoolchildren and teachers in Taiwan," Helicobacter, vol. 12, no. 3, pp. 258-264, 2007.

[9] L. Tsongo, J. Nakavuma, C. Mugasa, and E. Kamalha, "Helicobacter pylori among patients with symptoms of gastroduodenal ulcer disease in rural Uganda," Infection Ecology \& Epidemiology, vol. 5, 26785 pages, 2015.

[10] S. K. Chong, Q. Lou, M. A. Asnicar et al., "Helicobacter pylori infection in recurrent abdominal pain in childhood: comparison of diagnostic tests and therapy," Pediatrics, vol. 96, no. 2, pp. 211-215, 1995.

[11] A. M. R. De Oliveira, G. A. Rocha, D. M. De Magalhães Queiroz et al., "Evaluation of enzyme-linked immunosorbent assay for the diagnosis of Helicobacter pylori infection in children from different age groups with and without duodenal ulcer," Journal of Pediatric Gastroenterology and Nutrition, vol. 28, no. 2, pp. 157-161, 1999.

[12] C. Miller, "Power, land and ethnicity in the kassala-gedaref states: an introduction," in Land, Ethnicity and Political Legitimacy in Eastern Sudan, C. Miller, Ed., Cedej, Cairo, Egypt, 2005.

[13] S. Maleknejad, A. Mojtahedi, A. Safaei-Asl, Z. Taghavi, and E. Kazemnejad, "Primary antibiotic resistance to Helicobacter pylori strains isolated from children in northern Iran: A single center study," Iranian Journal of Pediatrics, vol. 25, no. 6, Article ID e2661, 2015.

[14] A. M. A. Telmesani, "Helicobacter Pylori: Prevalence and relationship with abdominal pain in school children in makkah city, western Saudi Arabia," Saudi Journal of Gastroenterology, vol. 15, no. 2, pp. 100-103, 2009.

[15] A. Çınar, M. Sadıç, H. İkbal Atılgan et al., "Prevalence of helicobacter pylori infection in school and pre-school aged children with C-14 urea breath test and the association with familial and environmental factors," Malecular Imaging and Radionuclide Therapy, vol. 24, no. 2, pp. 66-70, 2015.

[16] G. Ozbey, Y. Dogan, K. Demiroren, and I. H. Ozercan, "Prevalence of Helicobacter pylori in children in eastern Turkey and molecular typing of isolates," Brazilian Journal of Microbiology, vol. 46, no. 2, pp. 505-511, 2015.

[17] M. M. Biernat, B. Iwańczak, A. Bińkowska, J. Grabińska, and G. Gościniak, "The prevalence of helicobacter pylori infection in symptomatic children: A 13-year observational study in the lower silesian region," Advances in Clinical and Experimental Medicine, vol. 25, no. 2, pp. 303-308, 2016. 
[18] A. Eshraghian, "Epidemiology of Helicobacter pylori infection among the healthy population in Iran and countries of the Eastern Mediterranean Region: a systematic review of prevalence and risk factors," World Journal of Gastroenterology, vol. 20, no. 46, pp. 17618-17625, 2014.

[19] Y. Tindberg, T. H. Casswall, M. Blennow, C. Bengtsson, M. Granström, and M. Sörberg, "Helicobacter pylori eradication in children and adolescents by a once daily 6 -day treatment with or without a proton pump inhibitor in a double-blind randomized trial," Alimentary Pharmacology \& Therapeutics, vol. 20, no. 3, pp. 295-302, 2004.

[20] S. Koletzko, N. L. Jones, K. J. Goodman et al., "Evidence-based guidelines from ESPGHAN and NASPGHAN for Helicobacter pylori infection in children," Journal of Pediatric Gastroenterology and Nutrition, vol. 53, no. 2, pp. 230-243, 2011.

[21] M. A. Bin Mohanna, L. M. Al-Zubairi, and A. K. Sallam, "Prevalence of helicobacter pylori and parasites in symptomatic children examined for helicobacter pylori antibodies, antigens, and parasites in Yemen," Saudi Medical Journal, vol. 35, no. 11, pp. 1408-1411, 2014.

[22] E. Hestvik, T. Tylleskar, D. H. Kaddu-Mulindwa et al., "Helicobacter pylori in apparently healthy children aged 0-12 years in urban Kampala, Uganda: A community-based cross sectional survey," BMC Gastroenterology, vol. 10, article no. 62, 2010.

[23] D. M. Queiroz, J. G. Carneiro, M. B. Braga-Neto et al., "Natural History of Helicobacter pylori Infection in Childhood: EightYear Follow-Up Cohort Study in an Urban Community in Northeast of Brazil," Helicobacter, vol. 17, no. 1, pp. 23-29, 2012.

[24] A. Ceylan, E. Kırımi, O. Tuncer, K. Türkdogan, S. Ariyuca, and N. Ceylan, "Prevalence of helicobacter pylori in children and their family members in a district in Turkey," Journal of Health, Population and Nutrition, vol. 25, no. 4, pp. 422-427, 2007.

[25] Y. Tindberg, O. Nyrén, M. Blennow, and M. Granström, "Helicobacter pylori infection and abdominal symptoms among Swedish school children," Journal of Pediatric Gastroenterology and Nutrition, vol. 41, no. 1, pp. 33-38, 2005.

[26] M. A. Janjetic, P. Mantero, E. Cueto Rua et al., "Dietary and anthropometric indicators of nutritional status in relation to Helicobacter pylori infection in a paediatric population," British Journal of Nutrition, vol. 113, no. 7, pp. 1113-1119, 2015.

[27] K. Muhsen, S. Goren, and D. Cohen, "Helicobacter pylori infection in early childhood and growth at school age," Helicobacter, vol. 20, no. 6, pp. 410-417, 2015.

[28] J. Benson, R. A. Rahim, and R. Agrawal, "Newly arrived refugee children with Helicobacter pylori are thinner than their noninfected counterparts," Australian Journal of Primary Health, vol. 23, no. 1, pp. 92-96, 2017.

[29] M.-Y. Xu, L. Liu, B.-S. Yuan, J. Yin, and Q.-B. Lu, "Association of obesity with Helicobacter pylori infection: A retrospective study," World Journal of Gastroenterology, vol. 23, no. 15, 2750 pages, 2017.

[30] L. E. Bravo, R. Mera, J. C. Reina et al., "Impact of Helicobacter pylori infection on growth of children: A prospective cohort study," Journal of Pediatric Gastroenterology and Nutrition, vol. 37, no. 5, pp. 614-619, 2003.

[31] M. Kopacova, I. Koupil, B. Seifert et al., "Blood pressure and stature in Helicobacter pylori positive and negative persons," World Journal of Gastroenterology, vol. 20, no. 19, pp. 5625-5631, 2014. 


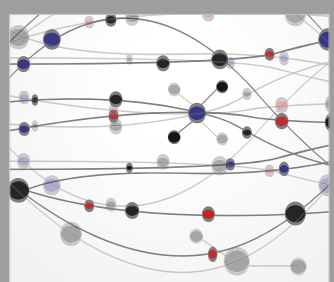

The Scientific World Journal
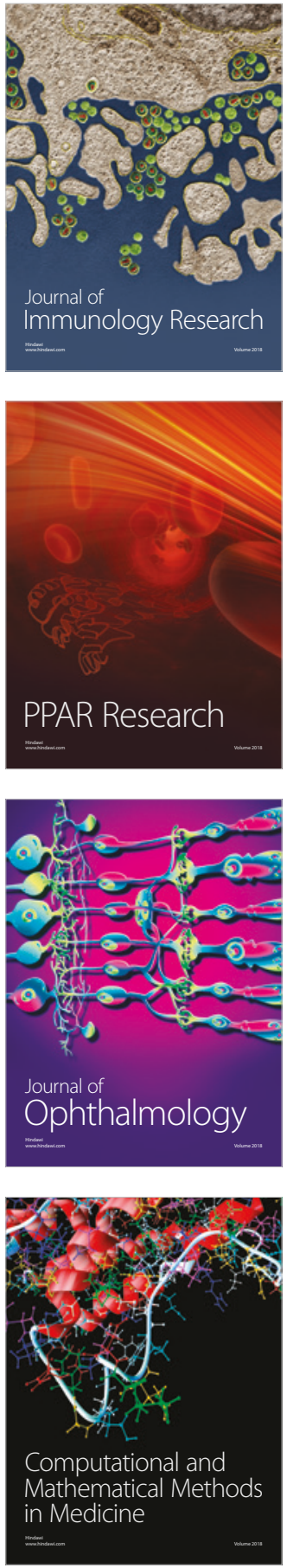

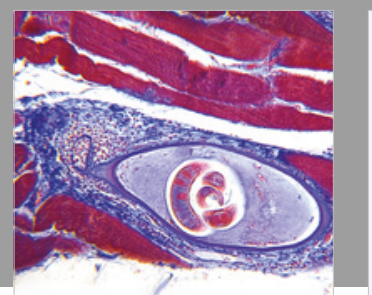

Gastroenterology Research and Practice

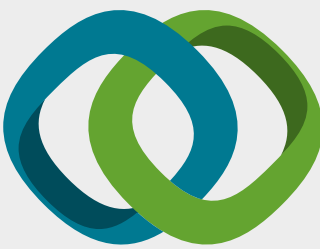

\section{Hindawi}

Submit your manuscripts at

www.hindawi.com
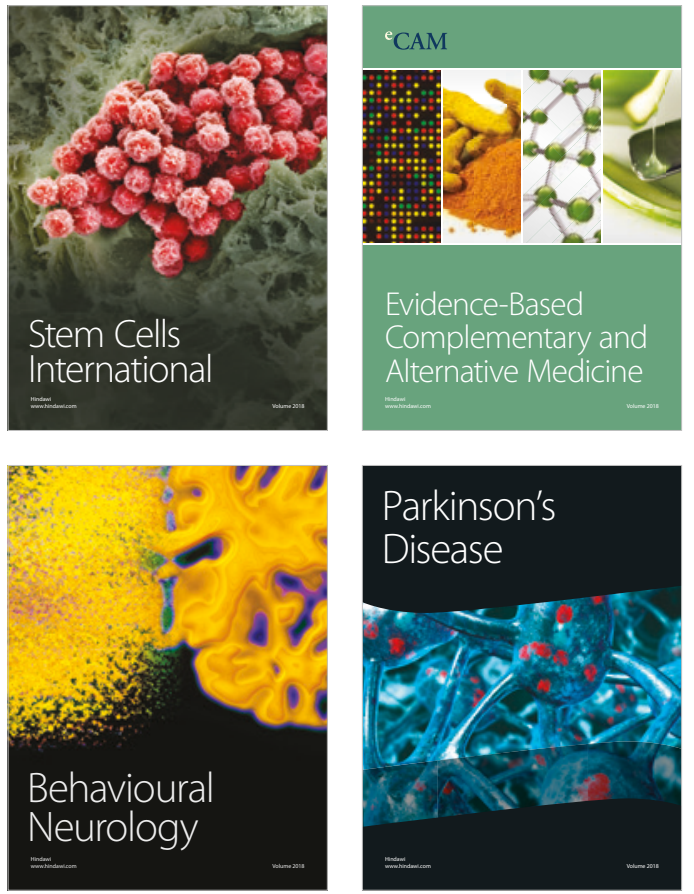

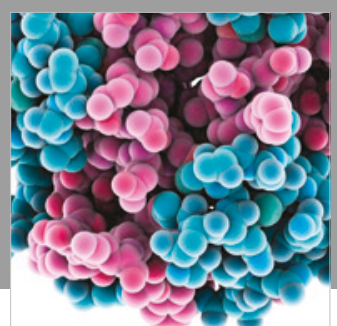

ournal of

Diabetes Research

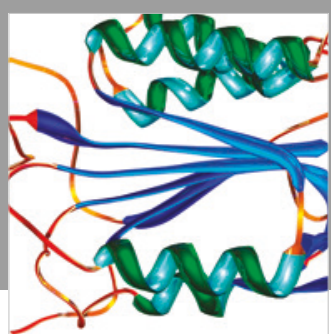

Disease Markers
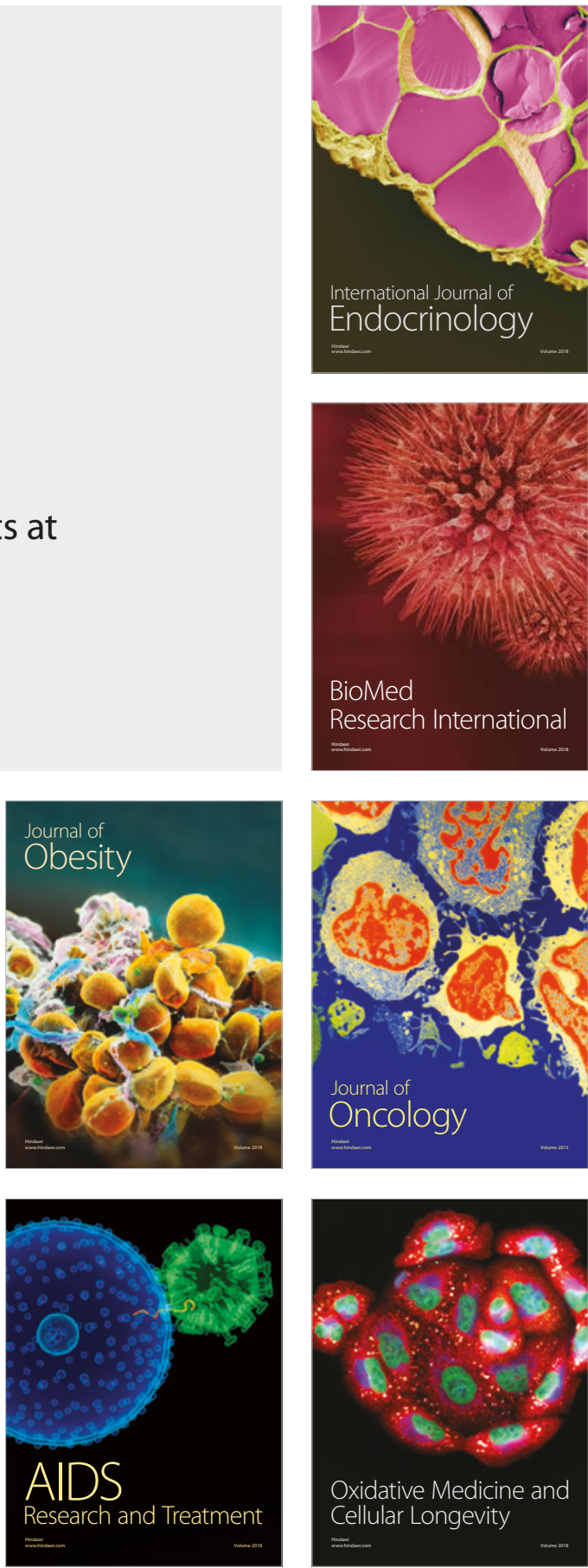